\title{
Production of knowledge: genetic basis, biochemical and immunological of Meares-Irlen Syndrome
}

\author{
Produção do conhecimento: bases genéticas, bioquímicas e \\ imunológicas da síndrome de Meares-Irlen
}

Fernanda Amaral Soares ${ }^{1}$, Lucília Silva Gontijo²

\begin{abstract}
Meares-Irlen syndrome, deficiency of the visual processing identified in individuals with dyslexia can be defined as a visual perceptive disorder that is believed to be originated in the primary visual cortex. This study deals with an integrative literature review aimed to analyze the scientific production in relation to biochemical, genetic and immunological basis that may be understood in this condition. Data were obtained via online query the databases of the Virtual Health Library (VHL) that interconnects several reference databases such as SciELO (Scientific Electronic Library Online), (Latin American and Caribbean Literature Rev Sciences health), MEDLINE (International Literature on Health Sciences), and the Digital Library of UFMG. A total of 16 articles, falling between 1996 and 2014 who met the inclusion criteria were included in the proposed study. Many studies have suggested an association of the syndrome with biochemical and genetic alterations. Some support the hypothesis that immune changes and metabolism of fatty acids are involved in cognitive and learning problems. Despite the scarce volume of studies on the subject, there is increasing evidence that there is a biochemical basis involved in a variety of visual disorders and learning.
\end{abstract}

Keywords: Dyslexia; Cholesterol; Perceptual disorders; Immunology; Genetics; Heredity

\section{RESUMO}

A Síndrome de Meares-Irlen, deficiência de processamento visual identificada em indivíduos com dislexia, pode ser definida como um distúrbio visual perceptivo que acredita ser originado no córtex visual primário. Este estudo consiste em uma revisão integrativa da literatura que teve como objetivo analisar a produção científica em relação às bases bioquímicas, genéticas e imunológicas que podem estar compreendidas nesta condição. Os dados foram obtidos por meio de consulta online às bases de dados da Biblioteca Virtual de Saúde (BVS) que interconecta várias bases de dados referenciais, como SciELO (Scientific Electronic Library Online), LILACS (Literatura Latino-Americana e do Caribe em Ciências da Saúde), MEDLINE (Literatura Internacional em Ciências da Saúde) e Biblioteca Digital da UFMG. Foram incluídos no estudo um total de 16 artigos, compreendidos entre os anos de 1996 e 2014 que atenderam aos critérios de inclusão propostos. Muitos estudos têm sugerido associação da síndrome com alterações bioquímicas e genéticas. Alguns sustentam a hipótese de que alterações imunológicas e no metabolismo de ácidos graxos estejam envolvidas com problemas cognitivos e de aprendizado. Apesar do escasso volume de estudos sobre o tema, existem evidências crescentes de que há uma base bioquímica envolvida em uma variedade de transtornos visuais e de aprendizagem.

Descritores: Dislexia; Colesterol; Transtornos da percepção; Imunologia; Genética; Hereditariedade

1 Graduation Course in Medicine, Faculdades Integradas Pitágoras de Montes Claros, Montes Claros, MG, Brazil.

${ }^{2}$ Faculdades Integradas Pitágoras de Montes Claros, Montes Claros, MG, Brazil.

The authors declare no conflicts of interests.

Received for publication 09/07/2014 - Accepted for publication 16/06/2015 


\section{INTRODUCTION}

I $\mathrm{n}$ recent years, researchers have identified a series of impairments in the visual processing of individuals with dyslexia. Meares-Irlen Syndrome falls into one of these impairments, and can be defined as a visual-perceptual disorder that is believed to be originated in the primary visual cortex ${ }^{(1)}$.

It is suggested that the syndrome is caused by excessive retinal sensitivity to specific frequencies of the spectrum of light. This sensitivity distorts the post-retinal processing, causing the electrical impulses to reach the cerebral cortex at different times, with loss of visual interpretation quality. It features a disruption in brain processing of information received by the visual system. These changes are not typically assessed by optometry examination. The symptoms described while reading include blur and presence of shadows on letters and words, duplication, movement, eye strain and fatigue ${ }^{(2)}$.

A new area of research aims at analyzing biochemical abnormalities and their implications for the deficits of visual processing and dyslexia. A variety of studies have suggested an association between essential fatty acids and visual problems in learning disabilities ${ }^{(3-5)}$.

Many findings associated to dyslexia, autism and other learning disorders are consistent with deficiency mainly in fatty acids omega 3 and omega 6. Polyunsaturated fatty acids (PUFA), especially the arachidonic acid (AA 20:4 n-6) and docosahexaenoic acid (DHA 22:6 n-3) are essential for human consumption because they are important components of the neuronal membranes, constituting $15-20 \%$ of the dry mass of the brain and more than $30 \%$ of the retina ${ }^{(3)}$.

AA and DHA also play an essential role in cellular processes related to learning and memory, and adequate levels of these fatty acids are essential for normal visual and cognitive development. Thus, abnormalities in fatty acid metabolism may be involved in cognitive and behavioral changes of these conditions, as well as on attention, language processing and difficulties associated to mood, digestion, sleep and temperature adjustment. In addition, reduced blood concentrations of longchain polyunsaturates fatty acids were found by several studies in individuals with Attention Deficit Hyperactivity Disorder (ADHD). Development disorders overlap with each other, being difficult to find a "pure" case. Thus, it is possible that this finding described for ADHD is also related to other such deficiencies as dyslexia, dyspraxia and autism ${ }^{(3)}$.

Some studies support the hypothesis that immunological and metabolical changes of AGPI are involved with the deficiency of these compounds in individuals with cognitive and learning problems. These conditions tend to group into families, and that evidence points to the possible existence of common genetic factors that may predispose the constitutional anomalies in the metabolism of fatty acids and phospholipids ${ }^{(2,3)}$..

From these literature findings, the aim of this study was to analyze the scientific studies regarding the biochemical, genetic and immunological bases that can be included in the MearesIrlen Syndrome.

\section{MethodS}

An extensive literature survey was conducted, which included an interactive review of the literature. The methodological design of the study was carried out with data collection from secondary sources.
The integrative review of the literature is a research method which allows the incorporation of evidence into clinical practice. This is a method ranging from the theoretical and empirical literature to the studies with different methodological approaches. For this, we use the results of research on a topic in a systematic and orderly manner, contributing to greater knowledge on the topic proposed ${ }^{(6)}$.

For access to articles in the sample of the present review we used the database from the Virtual Health Library (VHL), which organizes information in a structure that integrates and interconnects multiple reference databases, such as SciELO (Scientific Electronic Library Online), LILACS (Latin-American and Caribbean System on Health Sciences Information), MEDLINE (International Literature on Health Sciences), and UFMG Digital Library, consulted online on March 2014, using as descriptors: Dyslexia, Cholesterol, Perceptual Disorders, Immunology, Genetics, Heredity.

The descriptors were used in pairwise fashion, with 24 articles having been found in the search. All articles addressing the key words mentioned were included as a selection criteria, since they were available in full and mentioned Meares-Irlen Syndrome and its genetic, biochemical and immunological relations, and were published in Portuguese and in English. This way, 08 articles were deleted.

The year of publication was not cause of restriction in the choice of the articles due to the scarcity of recent literature on the topic discussed. The analysis of the data followed a questionnaire meeting the criteria for identifying authors, year of publication, title of magazine objectives of the research and content analysis, with the sample consisting of 16 quotations, 14 from articles and 02 from dissertations.

\section{RESULTS}

Of the 16 items used for the discussion of the topic proposed, $2(12.5 \%)$ were published in $1996,1(6.25 \%)$ in 2001 , $01(6.25 \%)$ in $2002,1(6.25 \%)$ in $2003,1(6.25 \%)$ in $2004,2(12.5 \%)$ in $2005,2(12.5 \%)$ in $2006,1(6.25 \%)$ in $2008,1(6.25 \%)$ in 2009,1 $(6.25 \%)$ in $2011,1(6.25 \%)$ in $2012,1(6.25 \%)$ in 2013 , and 1 $(6.25 \%)$ in 2014.

Regarding the publication language, 14 (87.5\%) were published in English and 02 (12.5\%) in Portuguese, and the articles in Portuguese from Brazil and the ones in English were 1 from Australia and the rest from the USA.

All the articles selected came from medical journals, and 11 (68.75\%) are about case-control studies, 2 (12.5\%) dissertations, and $3(18,75 \%)$ are literature reviews.

\section{DisCUSSION}

\section{Visual processing problems and dyslexia}

The Meares-Irlen Syndrome, a specific visual-perceptual impairment, has been increasingly accepted as a research area for visual problems that can be associated to dyslexia ${ }^{(2)}$.

In an attempt to elucidate its causes, there's a chance that there is an abnormal distribution of cones in the retina with the existence of extra peripherals cones and greater light sensitivity in peripheral vision. Due to the effort spent in processing visual information, reading becomes slower and segmented, producing fatigue, inversions and exchange of words, loss of lines in the 
text, loss of focus, drowsiness, visual disorders, headaches, irritability, nausea, distraction and photophobia, after a relatively short interval in reading, which compromises the speed of cognitive processing and memorization ${ }^{(2,7)}$.

The clinical features, such as the visual distortions of the printed words and the sense of movement or vibration of the text present in the Meares-Irlen Syndrome are common to the Visual Chronic Fatigue Syndrome, which may indicate an association between them. The symptoms described occur even in the absence of ophthalmologic abnormalities ${ }^{(8)}$.

Some studies have demonstrated benefits to reading with the use of colored transparencies on white paper for patients with Meares-Irlen Syndrome. Randomized clinical trials demonstrate that different people require different and specific colors, requiring that definition by the patients of the degree of $\operatorname{accuracy}^{(1,8,9)}$.

Despite the findings of improvement in reading with the use of colored filters, the causal basis to describe the visual symptoms and the reason why colored transparencies reduce these symptoms still need further research. Because of this, hypotheses about the biochemical bases related to the syndrome have been proposed ${ }^{(2,10)}$.

\section{Biochemical aspects related to the Syndrome of Meares-Irlen}

Some studies on the abnormal metabolism of fatty acids in people with dyslexia suggest that the visual processing can be affected by these disorders. Long-chain polyunsaturated fatty acids (PUFA) are important as structural components of the brain and the eyes, and are required for the normal functioning of the nervous system. There are two types of unsaturated fatty acids, omega- 6 and omega- 3 , and both can be converted into important essential fatty acids. Some of these fatty acids like eicosapentaenoic acid (EPA) and docosahexaenoic acid (DHA) make up about $15 \%$ to $30 \%$ of the dry weight of the retina, and are required for the structure, growth, remodeling and neuronal function, as well as play an essential role in cellular processes related to learning and memory. Thus, adequate levels of these fatty acids are essential for normal visual and cognitive development ${ }^{(2,3)}$.

A number of studies related to the abnormal metabolism of fatty acids in people with dyslexia suggests that visual processing can be affected by these anomalies, especially on Meares-Irlen Syndrome ${ }^{(2)}$.

A deficiency of essential fatty acids is significantly related to the onset of visual symptoms in reading, and even with some symptoms indicative of Meares-Irlen Syndrome as headache, sensitivity to light, movement and pulsation of words, and the halo effect around the words. Children with great clinical signs of fatty acid deficiency have significantly lower reading capability, and the severity of clinical signs is directly related to visual problems and symptoms during reading ${ }^{(11)}$.

Researchers have identified significant variations between blood lipids and urinary amino acids between a group of MearesIrlen Syndrome patients and the control group, which could be indicative of activation of the immune system of the patient ${ }^{(12)}$.

Findings from previous studies showed that the total plasma cholesterol values of Meares-Irlen Syndrome was significantly lower compared to the control group. On the other hand, there was an increase in the values of heptadecanoic acid found in the plasma of patients. The increased levels of heptadecanoic acid may involve changes in the membrane functions and in neurotransmission ${ }^{(5)}$.
There is some evidence suggesting that supplementation with essential fatty acids can improve neural function and the retina, which has been investigated as a possible cause of dyslexia and Meares-Irlen Syndrome ${ }^{(2)}$.

\section{Genetic predisposition and family nature}

Most research published is currently focused on the search for uni-causal solutions for dyslexia, emphasizing biological, environmental or cognitive factors. However, dyslexia and in particular the Meares-Irlen Syndrome is genetically heterogeneous, and can be caused by both genetic and environmental factors.

While investigating the family incidence of Meares-Irlen Syndrome in parents of children identified as carriers, researchers found $84 \%$ of incidence of symptoms similar to those of children. In this study, there was no distinction between data found for mothers and fathers. These findings suggest a possible genetic basis for visual processing deficits in patients with reading difficulties ${ }^{(13)}$.

Furthermore, the gene expression of the molecule carrier of apolipoprotein B100 lipids (APO-B100) has been correlated to abnormal metabolism of lipids, mainly related to plasma cholesterol levels. Cholesterol esters have proven importance in carrying essential fatty acids into the retina. The APO-B100encoding gene is located on the short arm of chromosome 2, and establishes close vicinity with DYX3 gene proven to confer susceptibility to dyslexia ${ }^{(14)}$.

The APO-B100 locus is recognized as one of the most polymorphic regions of the human genome. In a pilot study we found that certain variant alleles of APO-B100 were most commonly found in participants diagnosed with Meares-Irlen Syndrome than in those without this condition ${ }^{(14)}$.

\section{Relations to the immune system}

It has been suggested by some researchers that the construction of the metabolic profile of patients with MearesIrlen Syndrome could help develop more valid diagnostic categories and allow further investigation of disorders of the immune system as causal factors for learning and behavioral disorders ${ }^{(10,15-17)}$.

Some studies have shown differences in amino acid metabolism and lipid components in individuals with MearesIrlen Syndrome, and it indirectly supports the hypothesis that there is a biological and biochemical origin for this condition. Differences were found in the excretion of amino acids like proline, hydroxyproline and 3-methylhistidine, and this may result in dysregulation of the renewal of proteins and tissues. From this, it is speculated that this dysregulation may be related to immune system activation and cytokine production, which may have an effect on the genesis of visual dysfunction ${ }^{(10)}$.

There is evidence that dyslexic individuals and their families have a higher-than-normal incidence of autoimmune disorders. Evidence of this was suggested, showing that changes in the skill of reading were associated to the presence of signs indicative of infection evidenced by the finding of increased and sore lymph nodes $^{(10)}$.

Further investigation is still required to establish how changes in amino acids represent abnormal levels, confirm the relationship between biochemical anomalies identified and the metabolic functions that they represent, and also the association between these abnormalities and possible dysfunction of the immune system. 


\section{CONCLUSION}

Among the many causes that can lead to difficulties in reading, Meares-Irlen Syndrome remains little known, and its diagnosis can often be confused with developmental dyslexia. The study of this condition can help patients improve their difficulties with low-cost interventions, giving conditions for literacy and reading habits.

Many studies have focused on elucidating the causal factors of the Meares-Irlen Syndrome. This is important for new alternatives to be outlined in order to contribute to the visual development and learning their carriers, if possible, still during school literacy stage.

There is growing evidence that there is a biochemical base involved in a variety of visual and learning disorders, including the Meares-Irlen Syndrome, a subtype of dyslexia. Some questions remain unanswered, requiring more research to delineate the causal factors involved in this condition. Despite all the theories suggested for the Meares-Irlen Syndrome, its neurophysiological substrate is yet to be clarified.

\section{ACKNOWLEDGEMENTS}

The present research was developed with grant from Fundação de Amparo à Pesquisa do Estado de Minas Gerais FAPEMIG - Minas Gerais, Brazil.

\section{ReFERENCES}

1. Hollis J, Allen PM. Screening for Meares-Irlen Sensitivity in Adults: Can assessment methods predict changes in reading speed? Ophthal Physiol Opt. 2006; 26(6): 566-71.

2. Robinson GL, Sparkes DL, Roberts TK, Dustan RH, McGregor NR, Conway RNF. Biochemical anomalies in people with Irlen Syndrome: overlaping diagnostic categories, immune system disfunction and dietary Intervention [Tese]. Australia: University of Newcastle; 2002.

3. Richardson AJ. Long-chain polyunsaturated fatty acids in childhood developmental and psychiatric disorders. J Lipid Res. 2004; 39(12):1215-22.

4. Bell JG, Mackinlay EE, Dick JR, Macdonald DJ, Boyle RM, Glen ACA. Essential fatty acids and phospholipase A(2) in autistic spectrum disorders. FAB Research. 2004; 71(4):201-4.

5. Sparkes DL, Robinson GL, Dustan RH, Roberts TK. Plasma Cholesterol levels and Irlen Syndrome: Preliminary Study of 10- to 17- yr.-old students. Percept Mot Skills. 2003; 97(3):743-52.
6. Mendes KDS, Silveira RCP, Galvão CM. Revisão integrativa: Método de pesquisa para incorporação de evidências na saúde e na enfermagem. Texto Contexto Enferm. 2008; 17(4):758-64.

7. Faria LN. Frequência da Síndrome de Meares-Irlen entre alunos com dificuldades de leitura observadas no contexto escolar [Dissertação]. Belo Horizonte: Universidade Federal de Minas Gerais; 2011.

8. Loew SJ, Marsh NV, Watson K. Symptoms of Meares-Irlen/Visual Stress Syndrome in subjects diagnosed with Chronic Fatigue Syndrome. Internat J Clin Health Psychol. 2014; 14(2):87-92.

9. Loew SJ, Fernandés E, Watson K. Incidence of Meares-Irlen/ visual stress syndrome in reading and learning disorders: does fluorescent lighting in classrooms affect literacy and numeracy? Aula Abierta. 2013; 41(3):23-32.

10. Kriss I, Evans B. The relationship between dyslexia and MearesIrlen Syndrome. J Research Reading. 2005; 28(3):350-64.

11. Robinson GL, Roberts TK, McGregor NR, Dustan RH, Butt H. Understanding the causal mechanisms of visual processing problems: A possible biochemichal basis for Irlen Syndrome? Aust J Learn Disabil. 1999; 4(4):21-29.

12. Robinson GL, McGregor NR, Roberts TK, Dustan RH, Butt H. A biochemical analysis of people with chronic fatigue who have Irlen Syndrome: speculation concerning immune system disfunction. Percept Mot Skills. 2001; 93(2):486-504.

13. Robinson GL, Foreman PJ, Dear KBG. The familial incidence of symptoms of scotopic sensitivity/irlen syndrome. Percept Mot Skills. 1996; 83(3):1043-55.

14. Loew SJ, Watson K. A prospective genetic marker of the visualperception disorder Meares-Irlen Syndrome. Percept Mot Skills. 2012; 114(3):870-82.

15. Jones MG, Cooper E, Amjad S, Goodwin CS, Barron JL, Chalmers RA. Urinary and plasma organic acids and amino acids in chronic fatigue syndrome. Clin Chim Acta. 2005; 361(1-2):150-58.

16. Evans BJW, Wilkins AJ, Brown J, Busby A, Wingfield AE, Jeanes $\mathrm{R}$, et al. A preliminary investigation into the aetiology of MearesIrlen Syndrome. Ophthal Physiol Opt. 1996; 16(4):286-96.

17. Kruk R, Sumbler K, Willows D. Visual processing characteristics of children with Meares-Irlen Syndrome. Ophtal Physiol Opt. 2008; 28(1):35-46.

\section{Corresponding author:}

Lucília Silva Gontijo

Av. Prof. Aida Mainartina Paraiso, 80, Ibituruna, Montes Claros (MG), Brazil - ZIP Code: 39408-007.

Phone: (38) 9175-0009

E-mail: luciliagontijo@yahoo.com.br 